\title{
L'intervention éducative en sciences humaines et sociales au primaire à travers les zones d'ombre des discours sur les pratiques d'enseignement
}

Educational intervention in the social and human sciences at the primary level through the gray areas of official discourse on teaching practices

\section{La intervención educativa en ciencias humanas y sociales en primaria a través de las zonas grises de los discursos sobre las practicas educativas}

\author{
Anderson Araújo-Oliveira
}

Volume 47, numéro 2, automne 2019

Éduquer aux sciences humaines et sociales

URI : https://id.erudit.org/iderudit/1066453ar

DOI : https://doi.org/10.7202/1066453ar

Aller au sommaire du numéro

\section{Éditeur(s)}

Association canadienne d'éducation de langue française

ISSN

1916-8659 (numérique)

Découvrir la revue

\section{Citer cet article}

Araújo-Oliveira, A. (2019). L’intervention éducative en sciences humaines et sociales au primaire à travers les zones d'ombre des discours sur les pratiques d'enseignement. Éducation et francophonie, 47(2), 172-193.

https://doi.org/10.7202/1066453ar
Résumé de l'article

L’article présente une analyse du discours officiel relatif à l'enseignement des sciences humaines et sociales (SHS) au primaire. Pour ce faire, l'analyse descriptive des orientations gouvernementales sera accompagnée de l'identification d'un certain nombre de zones d'ombre (incohérences, ambiguïtés, contradictions, etc.) qui y sont sous-jacentes. Des apprentissages considérés comme étant importants à construire, mais qui ne font pas l'objet d'une évaluation, une compétence à développer considérée comme fondamentale alors qu'aucune plage horaire n'est prévue au régime pédagogique à son enseignement, des compétences centrées sur la capacité à raisonner et à argumenter alors que l'évaluation se centre davantage sur la description de connaissances factuelles n'en sont que quelques exemples. N'étant pas toujours univoques, ces orientations sont susceptibles de laisser place à des lectures et des interprétations variées sur le plan des paramètres devant présider à l'intervention éducative de l'enseignant, notamment en ce qui a trait à l'apport des SHS à la triple mission de l'école québécoise, au statut et à la place des savoirs scolaires au sein d'une approche par compétences, ainsi qu'au rôle de la démarche d'enseignement-apprentissage dans l'intervention éducative.

Tous droits réservés $@$ Association canadienne d’éducation de langue française, Ce document est protégé par la loi sur le droit d’auteur. L’utilisation des 2019 services d’Érudit (y compris la reproduction) est assujettie à sa politique d'utilisation que vous pouvez consulter en ligne.

https://apropos.erudit.org/fr/usagers/politique-dutilisation/ 


\title{
L'intervention éducative en sciences humaines et sociales au primaire à travers les zones d'ombre des discours sur les pratiques d'enseignement
}

\author{
Anderson ARAÚJ0-OLIVEIRA
}

Université du Québec à Montréal, Montréal, Québec

\section{RÉSUMÉ}

L'article présente une analyse du discours officiel relatif à l'enseignement des sciences humaines et sociales (SHS) au primaire. Pour ce faire, l'analyse descriptive des orientations gouvernementales sera accompagnée de l'identification d'un certain nombre de zones d'ombre (incohérences, ambiguïtés, contradictions, etc.) qui y sont sousjacentes. Des apprentissages considérés comme étant importants à construire, mais qui ne font pas l'objet d'une évaluation, une compétence à développer considérée comme fondamentale alors qu'aucune plage horaire n'est prévue au régime pédagogique à son enseignement, des compétences centrées sur la capacité à raisonner et à argumenter alors que l'évaluation se centre davantage sur la description de connaissances factuelles n'en sont que quelques exemples. N'étant pas toujours univoques, ces orientations sont susceptibles de laisser place à des lectures et des interprétations variées sur le plan des paramètres devant présider à l'intervention éducative de l'enseignant, notamment en ce qui a trait à l'apport des SHS à la triple mission de l'école québécoise, au statut et à la place des savoirs scolaires au sein d'une approche par 
L'intervention éducative en sciences humaines et sociales au primaire à travers les zones d'ombre des discours sur les pratiques d'enseignement

compétences, ainsi qu'au rôle de la démarche d'enseignement-apprentissage dans l'intervention éducative.

\title{
ABSTRACT
}

\section{Educational intervention in the social and human sciences at the primary level through the gray areas of official discourse on teaching practices}

Anderson ARAÚJ0-OLIVEIRA, University of Quebec in Montreal, Montreal, Quebec

The article presents an analysis of the official discourse on teaching social and human sciences (SHS) at the primary level. The descriptive analysis of government orientations will be accompanied by the identification of some gray areas (inconsistencies, ambiguities, contradictions, etc.) underlying them. A few examples are knowledge considered important to build on but not subject to evaluation, essential skills that need to be developed but are given no teaching time slot in the educational system, skills that rely on the ability to reason and make arguments, while the evaluation focuses more on the description of factual knowledge. Not always clear, these orientations can leave room for different interpretations and readings of the parameters that should govern the teacher's educational intervention, especially regarding the contribution of SHS to the triple mission of Quebec schools to the status and the place of school knowledge within a skills-based approach, and the role of the teaching-learning approach in the educational intervention.

\section{RESUMEN}

\section{La intervención educativa en ciencias humanas y sociales en primaria a través de las zonas grises de los discursos sobre las practicas educativas}

\author{
Anderson ARAÚJ0-OLIVEIRA, Universidad de Quebec en Montreal, Montreal, Quebec
}

El artículo presenta un análisis del discurso oficial relacionado con la enseñanza de las ciencias humanas y sociales (SHS) en primaria. A tal efecto, el análisis descriptivo de las orientaciones gubernamentales estar acompañado de la identificación de un cierto número de zonas grises (incoherencias, ambigüedades, contradicciones, etc.) que se encuentran subyacentes. Aprendizajes que se consideran importantes de construir, pero que no están sujetos a una evaluación; una competencia por desarrollar considerada como fundamental pero a la que no se le ha reservado rango de tiempo en el régimen pedagógico para ser enseñada; competencias centradas en la capacidad de razonar y de argumentar pero cuya evaluación se centra sobre todo sobre la descripción de conocimientos factuales, no son sino algunos ejemplos. Como nunca son unívocas, dichas orientaciones son susceptibles de dar cabida a la 
lectura y a interpretaciones variadas en lo referente a los parámetros que deben regir la intervención educativa del maestro, especialmente en lo referente a la aportación de las SHS a la triple misión de la escuela quebequense, al estatus y al lugar de los saberes escolares en el seno de un enfoque por competencias, así como el rol del procedimiento de la enseñanza-aprendizaje en la intervención educativa.

Dix-sept ans après la mise en œuvre d'une refonte importante de l'enseignement des sciences humaines et sociales (SHS) au primaire au Québec, et de la publication de nombreux documents qui complètent et clarifient les orientations ministérielles, un constat s'impose: du Programme de formation de l'école québécoise (Gouvernement du Québec, 2001) à la Progression des apprentissages (Gouvernement du Québec, 2009), en passant par le Renouveau pédagogique (Gouvernement du Québec, 2005a), le Cadre d'évaluation des apprentissages (Gouvernement du Québec, 2011), l'Évaluation du matériel didactique (Gouvernement du Québec, 2004, 2007), les acteurs et actrices du monde de l'éducation (enseignants et enseignantes, conseillers et conseillères pédagogiques, concepteurs et conceptrices de manuels scolaires, chercheurs et chercheures, formateurs et formatrices universitaires, futurs enseignants et futures enseignantes, etc.) sont confrontés à un ensemble de discours sur les pratiques d'enseignement ${ }^{1}$ en SHS qui ne sont pas toujours univoques, et qui sont traversés par des sous-entendus, des incohérences, des ambiguïtés et des contradictions que l'on appellera ici les zones d'ombre ${ }^{2}$ des discours sur les pratiques d'enseignement.

Sur la base des réflexions issues de nos recherches portant sur l'étude des pratiques d'enseignement réalisées au cours des 15 dernières années, cet article présente une analyse critique du discours officiel relatif à l'enseignement des SHS au primaire. Cet article ne prétend pas reprendre les conclusions de ces recherches, mais simplement mettre en exergue un certain nombre de tensions véhiculées dans la documentation gouvernementale entourant l'apport des SHS à la triple mission de l'école québécoise, le statut et la place des savoirs scolaires disciplinaires au sein d'une approche par compétences, ainsi que l'apport et le rôle de la démarche d'apprentissage.

Le corpus qui a fait l'objet de l'analyse est constitué de onze documents officiels publiés par le Gouvernement du Québec de 1996 à 2016, soit dans le contexte du renouvellement des programmes de formation au primaire, et présentant un ou

1. Par discours sur les pratiques d'enseignement, il faut entendre ici l'ensemble des documents officiels produits par des instances mandatées qui légifèrent, prescrivent, encadrent et orientent l'actualisation des pratiques des enseignants.

2. Dans ce texte, il faut comprendre par zones d'ombre un ensemble de sous-entendus, d'incohérences, d'ambiguîtés ou de contradictions pouvant être dégagés du discours officiel relatif à l'enseignement des SHS et susceptibles de laisser place à des interprétations variées sur le plan des paramètres devant présider à l'intervention éducative de l'enseignant. 
plusieurs liens avec l'enseignement des sciences humaines et sociales au primaire. Il s'agit essentiellement du Programme de formation de l'école québécoise, des énoncés de politique éducative qui l'ont précédé (Rapport final de la commission des États généraux sur l'éducation, Plan d'action pour la réforme de l'éducation, etc.) et des orientations complémentaires qui lui ont succédé (Évaluation des aspects pédagogiques du matériel éducatif, Cadre d'évaluation des apprentissages, etc.). Les documents qui ont fait l'objet de l'analyse sont précédés d'un astérisque $\left({ }^{*}\right)$ dans la liste des références bibliographiques.

La collecte des données s'est effectuée au moyen d'une grille d'analyse thématique élaborée au fil du temps en fonction des questions spécifiques découlant des contextes, des problématiques et des cadres conceptuels sous-jacents aux différentes recherches réalisées par l'auteur de ce texte au cours des 15 dernières années, et qu'il est possible de visualiser dans le tableau suivant (Tableau 1).

\section{Tableau 1. Grille d'analyse}

1. Pourquoi enseigne-t-on les SHS au primaire (finalités)?

- Apports des SHS à la mission d'instruction

- Apports des SHS à la mission de socialisation

- Apports des SHS à la mission de qualification

2. Qu'enseigne-t-on en SHS au primaire (objets d'apprentissage et d'évaluation)?

2.1 Vision constructiviste de l'apprentissage

- Statut des savoirs disciplinaires

- Modalités d'accession aux savoirs

- Place et rôle de l'élève

- Place et rôle de l'enseignant

2.2 Approche par compétences

- Place des savoirs disciplinaires

- Rôle des savoirs disciplinaires

3. Comment enseigne-t-on les SHS au primaire (démarches d'apprentissage)?

- Structure de la démarche de recherche

- Place et rôle des savoirs disciplinaires

- Rôle de la démarche de recherche

- Place et rôle de l'élève

- Place et rôle de l'enseignant

4. Autres

Dans les pages qui suivent, la description des orientations ministérielles sera accompagnée de l'identification des zones d'ombre qui y sont sous-jacentes. Des apprentissages identifiés comme étant importants à construire, mais qui ne font pas l'objet d'une évaluation systématique, une compétence à développer alors qu'aucune plage 
horaire n'est prévue au régime pédagogique à son développement, des compétences centrées sur la capacité à raisonner et à argumenter alors que l'évaluation se centre davantage sur la description de connaissances factuelles n'en sont que quelques exemples. Du surcroît, n'étant pas toujours univoques, les orientations ministérielles sont susceptibles de laisser place à des interprétations variées sur le plan des paramètres devant présider à l'intervention éducative de l'enseignant.

\section{ZONE D'OMBRE 1: LES SHS AU PRIMAIRE ET LA TRIPLE MISSION ÉDUCATIVE DE L'ÉCOLE}

L'école québécoise «a le mandat de préparer l'élève à contribuer à l'essor d'une société voulue démocratique et équitable» (Gouvernement du Québec, 2001, p. 2). Ainsi, si sa responsabilité première renvoie au développement intellectuel des élèves, elle ne demeure pas la seule, car l'école se voit également mandatée pour contribuer à l'insertion harmonieuse des jeunes dans la société à laquelle ils appartiennent en leur permettant de s'approprier les savoirs et les valeurs qui la fondent et en les formant pour qu'ils soient en mesure de participer à son évolution. Or, alors que les programmes précédents étaient marqués par le sceau d'une perspective identitaire en privilégiant le développement du sentiment d'appartenance, dans le cadre du programme actuel, l'enseignement des SHS doit concourir à la réalisation de la triple mission de l'école québécoise. La mission d'instruction vient «réaffirmer l'importance de soutenir le développement cognitif aussi bien que la maîtrise des savoirs" (Ibid., p. 3), alors que celle de socialisation contribue «à l'apprentissage du vivre ensemble et au développement d'un sentiment d'appartenance à la collectivité» (Ibid.). La mission de qualification, quant à elle, renvoie à la nécessité d'offrir aux élèves les conditions les plus adéquates possible pour entreprendre et réussir leur parcours scolaire, en plus de renvoyer aussi à la nécessité de rendre les élèves aptes à participer au développement de la société par l'intégration professionnelle (Ibid.).

Or, bien que le discours officiel soutienne depuis longtemps que l'école représente le lieu par excellence d'un apprentissage guidé qui permet à l'élève d'acquérir les connaissances, les habiletés et les attitudes nécessaires pour comprendre et transformer le monde et pour continuer à apprendre tout au long de la vie (Gouvernement du Québec, 1996, 1997a, 1997b), il faut souligner que la réflexion entourant la triple mission attribuée à l'école reste pour le moins partielle (on ne lui accorde qu'une demi-page sur les 350 qui composent la version officielle du Programme de formation) et très peu articulée autour des caractéristiques particulières des programmes disciplinaires. En ce sens, encore qu'il nous soit possible de faire quelques inférences 
à partir de notre propre cadre conceptuel ${ }^{3}$, il faut reconnaitre que la façon dont chacune des disciplines, dans la spécificité des finalités éducatives, des savoirs et des dispositifs de formation qui leur sont sous-jacents, contribuera à la réalisation de cette triple mission reste peu explicitée dans les documents ministériels (Araújo-Oliveira, 2010). Comment l'enseignement des SHS contribuerait-il au développement de la mission d'instruction, par exemple? Serait-ce par le développement chez les élèves de concepts historiques ou géographiques et d'habiletés intellectuelles? Ou serait-ce plutôt par l'apprentissage des valeurs socialement acceptables, telles la solidarité, la justice, la coopération, etc.? Dans quelle mesure cet enseignement peut-il contribuer effectivement à chacune des missions préconisées? Ne privilégierait-il pas l'une des missions plutôt qu'une autre? Pourrait-on socialiser ou qualifier sans instruire, et vice-versa?

Certes, l'orientation actuelle au sein de la didactique des SHS au primaire conduit à privilégier sur le plan de ses finalités un certain équilibre entre l'instruction, la socialisation et la qualification en mettant en évidence le caractère praxéologique de cette discipline, en ce sens qu'il faut apprendre les SHS pour comprendre le monde et éventuellement pour y agir (Laville, 1991; Lenoir, 1989; Libresco, 2003). Néanmoins, la primauté accordée depuis longtemps par cet enseignement à la transmission d'un corpus de savoirs sur la société d'appartenance des élèves pour permettre l'apprentissage du vivre ensemble et la formation de la personne (Audigier, 1999) amène à se questionner sur les possibilités des enseignants de prendre réellement en compte l'équilibre souhaité et, ainsi, contribuer au développement de la triple mission. De plus, l'adhésion du nouveau programme à une approche par compétences fortement associée à une politique néolibérale (Bronckart et Dolz, 2002) (rôle limité de l'État en matière économique et sociale, ouverture à la loi du marché, vision de l'individu en tant que capital humain, etc.) conduit à se demander si l'une des finalités ne serait pas davantage valorisée au détriment des deux autres, ou encore si elles ne seraient pas simplement réduites à quelques-unes de leurs dimensions.

3. De notre point de vue, en SHS, la mission d'instruction se traduit par le développement de conceptions ainsi que d'habiletés et d'attitudes intellectuelles, de même que par l'acquisition et l'application d'une démarche systématique et rigoureuse. De son côté, le développement d'un sentiment d'appartenance à la collectivité caractéristique de la mission de socialisation requiert l'appropriation du patrimoine culturel, traduit en termes de savoirs scientifiques et culturels produits par l'humanité, puis l'acquisition des valeurs à la base de la société (solidarité, justice, coopération, démocratie, etc.) et l'exercice de la citoyenneté par la mobilisation des savoirs disciplinaires dans la vie quotidienne (Lebrun, Hasni et Oliveira, 2008). Enfin, la qualification renvoie à la nécessité de cette discipline d'offrir aux élèves les bases nécessaires pour réussir un parcours scolaire, de même que la nécessité de les rendre aptes à s'intégrer à la société par le développement et la mobilisation des compétences acquises dans des situations diverses en lien avec la vie professionnelle. 


\section{ZONE D'OMBRE 2: LES SHS AU PRIMAIRE ET LA VISION CONSTRUCTIVISTE DE L'APPRENTISSAGE}

Contrairement aux programmes antérieurs qui se fondaient soit sur une approche humaniste (Gouvernement du Québec, 1971), soit sur une approche par objectifs comportementaux d'inspiration néo-béhavioriste (Gouvernement du Québec, 1981, 1983), le programme actuel, tout en reconnaissant l'apport du béhaviorisme dans les apprentissages, revendique ouvertement une approche constructiviste ${ }^{4}$ (Gouvernement du Québec, 2001). Selon cette approche, le développement cognitif est un processus par lequel l'être humain reconstruit la culture du groupe social auquel il appartient (Coll, Martín, Mauti et Miras, 1993). Dans cette perspective, la connaissance résulte d'un complexe processus de modification, de réorganisation et de construction mis en place par les élèves pour assimiler et interpréter la réalité naturelle, humaine et sociale.

Envisagée du point de vue de l'enseignement des SHS, cette approche préconise que l'apprentissage ne soit jamais un rapport direct et immédiat d'appropriation de la réalité humaine et sociale par le sujet apprenant. Elle passe par le détour nécessaire d'un système objectif de régulation, lui-même devant faire l'objet d'un processus de construction résultant de la rupture que le sujet établit avec l'objet en se l'appropriant (Lenoir, 1996). Cette acception consiste, d'un côté, à considérer l'appréhension de la réalité humaine et sociale comme le résultat d'une production humaine socialement et historiquement située (Lenoir, 1993; Tutiaux-Guillon, 2015; Werneck, 2006) et, de l'autre, à exclure l'idée d'une réalité indépendante, d'un savoir préexistant historique ou géographique transmis par héritage ou encore disposé dans le réel et attendant d'être découvert par le sujet (Castañon, 2007; Gaya, 2006). Le savoir constitue les outils à partir desquels l'élève sera en mesure d'organiser son expérience empirique, de développer sa pensée réflexive, sa conscience critique et, par le fait même, de pouvoir agir de façon réfléchie sur le monde qui l'entoure.

La perspective constructiviste octroie à l'élève un rôle actif dans la réalisation de sa vision du monde, puisqu'il est responsable de la production de la réalité humaine et sociale. Dans une telle perspective, "l'apprentissage est considéré comme un processus dont l'élève est le premier artisan" (Gouvernement du Québec, 2001, p. 5) et un tel apprentissage n'est possible qu'au moyen de situations d'enseignement-apprentissage qui représentent un réel défi pour l'élève, c'est-à-dire «des situations qui entraînent une remise en question de ses connaissances et de ses représentations personnelles» (Ibid.). Autrement dit, c'est l'élève qui construit, modifie et enrichit ses schémas cognitifs.

4. "Certains apprentissages que doit développer l'école bénéficient de pratiques d'inspiration béhavioriste axées, notamment, sur la mémorisation de savoirs au moyen d'exercices répétés. Cependant, beaucoup d'éléments du Programme de formation, en particulier ceux qui concernent le développement de compétences et la maîtrise de savoirs complexes, font appel à des pratiques basées sur une conception de l'apprentissage d'inspiration constructiviste. » (Gouvernement du Québec, 2001, p. 5.) 
Puisque l'accession au savoir est vue comme une production de la part du sujet apprenant, comme un processus d'objectivation médiatisé par une démarche structurée et rigoureuse, l'enseignement des SHS doit dépasser la fonction patrimoniale qui privilégie le développement du sentiment d'appartenance à la société au sein de laquelle il s'est façonné. Ces disciplines exigent beaucoup plus que la mémorisation de dates, de noms de personnages et de lieux historiques. Sans oblitérer le nécessaire développement du sentiment identitaire, elles requièrent plutôt le développement de la capacité de questionner, de raisonner, d'analyser - autrement dit, le développement d'une attitude critique et réflexive à l'égard des phénomènes humains et sociaux survenus dans le temps et dans l'espace. En conséquence, l'enseignant a pour fonction de promouvoir la réalisation du meilleur apprentissage possible (Coll et al., 1993). Comme le dit Lenoir (1989, p. 684), son rôle consiste à

favoriser l'interaction entre le sujet et l'objet, [à] intervenir au niveau du processus d'apprentissage lui-même, pour ce qui est de la planification, de l'orientation, du support et de l'évaluation des apprentissages en cours, non à se substituer au sujet dans une perspective de révélation d'un savoir vérité.

Or il faut noter que faire appel au constructivisme comme fondement du processus enseignement-apprentissage ne garantit pas que les orientations, du point de vue de leur opérationnalisation, aient réellement évolué. D'une part, la reconnaissance de l'apport du béhaviorisme donne l'impression que l'appréhension de la réalité sociale et humaine peut se faire en tant que modification d'un comportement, conséquence d'une réponse à des stimuli, plutôt qu'en termes d'interaction et d'objectivation entre l'individu et le réel (Araújo-Oliveira, 2010). D'autre part, plusieurs auteurs (Coll, 2003; Werneck, 2006) soulignent l'existence d'une grande polysémie autour du terme constructivisme. Il renvoie à une acception tantôt psychologique, tantôt épistémologique ou didactique. Dans son acception psychologique, par exemple, le terme constructivisme emprunte soit au constructivisme piagétien basé sur la psychologie génétique, soit au cognitivisme centré sur le traitement de l'information, ou encore au constructivisme socioculturel inspiré de la théorie historique et socioculturelle. Comme aucune source n'est citée pour permettre de comprendre exactement quelle conception du constructivisme sous-tend le discours officiel, l'interprétation peut varier fortement et ainsi constituer un obstacle à son utilisation en classe.

De plus, selon Lenoir et Larose (2005), la perspective constructiviste, lorsque mal interprétée, peut être facilement associée à des procédures d'enseignement plaçant l'élève au cœur des apprentissages scolaires par le recours à ses préconceptions et aux interactions sociales avec des pairs, ce qui amènerait à négliger, entre autres, les savoirs à enseigner. Une telle vision peut éventuellement justifier des pratiques d'enseignement en SHS spontanéistes axées sur le laisser-faire, dans la mesure où elles surestiment la capacité des sujets. Elles risquent aussi de minimiser la dimension didactique de l'intervention éducative et de considérer cette dernière 
comme secondaire, en ce sens qu'elles sous-estiment le rôle de l'enseignant en tant que médiateur externe du rapport cognitif que l'élève établit avec les objets d'apprentissage.

\section{ZONE D'OMBRE 3: LES SHS AU PRIMAIRE ET L'APPROCHE PAR COMPÉTENCES}

Si, dans le programme par objectifs (Gouvernement du Québec, 1981, 1983), l'enseignement des SHS visait l'acquisition de connaissances et, surtout, de certaines habiletés comportementales (savoir-faire et savoir-être), il vise maintenant le développement de compétences disciplinaires fondées sur la construction des savoirs dits «essentiels». Il semble que la définition que Perrenoud (2002) donne à la compétence est reprise presque mot pour mot par le Ministère, sans en identifier la source. La compétence renvoie à la capacité de l'élève de choisir, de mobiliser et de combiner des ressources diverses pour répondre à des situations complexes et singulières. Ces ressources font référence à un ensemble d'acquis qui ont en commun le fait de pouvoir être mobilisables lorsque le sujet affronte des situations nouvelles (Jonnaert, Barrette, Boufrahi et Masciotra, 2004; Perrenoud, 2002). En ce sens, les savoirs disciplinaires sont considérés comme des ressources essentielles tant au développement des compétences qu'à leur exercice. L'enseignement des SHS doit ainsi participer au développement de cette capacité de mobiliser des savoirs divers pour répondre à des situations complexes et singulières.

$\mathrm{Au} 1^{\mathrm{er}}$ cycle, la compétence s'énonce ainsi: «construire sa représentation de l'espace, du temps et de la société» (Gouvernement du Québec, 2001, p. 166). Elle consiste d'une part à apprendre «à lire et à visualiser une réalité spatiale ou temporelle et à exprimer la durée de diverses façons» et, d'autre part, à «découvrir qu'un groupe, quel qu'il soit, possède des caractéristiques et un fonctionnement particuliers" (Ibid.). Son développement repose sur l'étude de différents groupes sociaux, auxquels l'élève participe, et des éléments naturels et humains des paysages en vue de dégager des ressemblances, des différences et des changements survenus, et il repose aussi sur l'initiation aux principales techniques relatives au temps et à l'espace (ligne du temps, calendrier, plan, maquette, etc.). Aux $2^{\mathrm{e}}$ et $3^{\mathrm{e}}$ cycles, trois compétences sont visées. La première porte sur une analyse de l'organisation sociospatiale d'une société donnée à un moment précis de son histoire, soit du point de vue de sa localisation, de ses caractéristiques, de l'influence de certains personnages ou de l'incidence de certains événements sur son organisation. La deuxième implique d'analyser une même société et son territoire à deux moments différents de son histoire, de façon à identifier les changements survenus (mode d'occupation du territoire, démographie, peuplement, etc.), ainsi que les événements et personnages ayant eu une certaine influence sur ces changements. Enfin, la troisième porte sur l'analyse de l'organisation sociospatiale de deux sociétés distinctes à un même moment historique en 
mettant en évidence leurs différences et leurs ressemblances sur divers aspects (activités économiques et culturelles, composition et répartition de la population, etc.).

Ces compétences requièrent que les élèves dépassent la description des réalités sociales et territoriales pour se lancer dans l'interprétation, dans la mesure où elles insistent sur l'analyse distancée des sociétés et de leur territoire sous trois angles différents: l'organisation, le changement et la diversité. Cette nouvelle configuration s'inscrit dans la perspective prônée depuis plusieurs années par les didacticiens des SHS, laquelle sous-entend que l'enseignement de cette discipline vise le développement de la pensée critique, de l'autonomie intellectuelle, et de la capacité de questionnement, de raisonnement, d'analyse, de synthèse, de jugement critique, etc., dans l'étude du phénomène humain plutôt que la mémorisation d'un ensemble de données factuelles décontextualisées (ex.: Daudel, 1990; Laurin, 1998; Lebrun, 1993; Le Roux, 2005; Moniot, 1993).

D'un point de vue opérationnel, alors qu'au $1^{\text {er }}$ cycle le développement de la compétence s'appuie sur l'étude de différents groupes sociaux et de techniques relatives au temps et à l'espace, aux $2^{\mathrm{e}}$ et $3^{\mathrm{e}}$ cycles ce développement doit s'appuyer sur l'étude et la compréhension des dynamiques sociospatiales de diverses sociétés prédéterminées par le programme. Toutefois, comme le souligne Lebrun (2009), ces sociétés ne représentent que le «terrain d'exercice» où l'élève développera progressivement son système d'interprétation systématique et rigoureuse des réalités humaines, c'est-àdire la grille d'analyse à partir de laquelle il pourra décrire, interpréter et découvrir la diversité de différentes sociétés. La construction de cette grille présuppose deux opérations distinctes, mais interreliées (Ibid.). La première consiste à dégager, d'un point de vue descriptif, les caractéristiques, les composantes et la dynamique fonctionnelle des sociétés étudiées (ex.: la Nouvelle-France vers 1645, les Incas vers 1500, etc.). La seconde, qui ne peut se réaliser qu'à partir des résultats obtenus à la première, renvoie d'abord à l'identification des principaux changements survenus dans une société et sur son territoire à deux moments distincts - lecture diachronique - (ex.: la Nouvelle-France entre 1645 et 1745) ainsi qu'à la construction d'une explication argumentée et justifiée quant aux causes et conséquences de ces changements. Elle renvoie également à la reconnaissance des principales différences et ressemblances entre deux sociétés distinctes et leurs territoires à une même époque - lecture synchronique - (ex. : les Inuits et les Micmacs vers 1980) ainsi qu'à la construction d'une explication argumentée quant aux causes et aux conséquences des différences.

L'adhésion à une approche par compétences en enseignement des SHS donne malheureusement lieu à de nombreuses lacunes. D'une part, comme le remarque Caillot (1994), il s'agit d'un concept non stabilisé qui n'avait pas fait l'objet d'une analyse systématique approfondie avant d'être introduit dans les programmes de formation au primaire. En ce sens, «l'introduction de cette approche présente des risques, autant par l'absence de véritables réflexions sur ses fondements épistémologiques et théoriques que par la mainmise annoncée d'une logique marchande et économique 
sur le monde de l'éducation» (Jonnaert et al., 2004, p. 668). D'autre part, la polysémie sémantique de la notion de compétence présente dans la documentation scientifique (Caillot, 1994; Lenoir et al., 1999), et qui trouve son prolongement dans la documentation gouvernementale (Jonnaert, 2002; Larose, Lenoir, Pearson et Morin, 2005), est susceptible de générer différentes perspectives d'articulation entre les savoirs disciplinaires prévus et les compétences à développer en SHS, comme le mettent en évidence Lebrun et Araújo-Oliveira (2010), selon qu'on interprète cette notion en fonction de l'un ou l'autre des modèles de compétence ${ }^{5}$. Selon leurs réflexions, en considérant les compétences selon la perspective de la cognitive constructs of competence, c'est-à-dire en tant que pouvoir d'action que posséderait l'être humain d'adapter ses paroles et ses actions à une infinité de situations inédites, l'enseignement des SHS privilégierait davantage le développement intellectuel et critique de l'élève, mais sans négliger pour autant le développement des connaissances techniques et patrimoniales. Par contre, lorsqu'elles sont comprises selon un modèle de la behaviorist competence en tant que comportement attendu (réponse adéquate) en fonction de certaines conditions introduites par l'enseignant, ou encore selon le modèle de la generic competence vu comme un comportement coordonné et organisé dans une action finalisée, l'enseignement de cette discipline reposerait plutôt sur la maîtrise d'habiletés techniques et de connaissances factuelles telles la lecture de cartes, la capacité à s'orienter, la connaissance des institutions et de leur évolution, etc. En ce sens, ainsi que l'indique Jonnaert (2006), comme la conceptualisation de la notion de compétence ainsi que les moyens de l'opérationnaliser et de l'évaluer restent, dans le cadre de la documentation ministérielle, un point de vue nébuleux, il n'est guère possible de la clarifier et, par conséquent, de l'utiliser de façon adéquate dans les pratiques en milieu scolaire. De plus, puisque le programme d'étude ne spécifie pas explicitement l'ensemble des apprentissages visés (concepts clés, habiletés intellectuelles, attitudes intellectuelles, etc.), le choix des apprentissages que les élèves doivent réaliser afin de développer les compétences préconisées et assurer leur réutilisation adéquate dans des contextes extrascolaires non disciplinaires demeure du ressort des enseignants. Or cette configuration disciplinaire soulève ainsi de nombreuses interrogations, notamment sur les possibilités de son opérationnalisation en contexte réel d'intervention éducative. À partir de quels paramètres les enseignants, qui ont à actualiser ces orientations dans leurs pratiques, effectuent-ils le choix des apprentissages qui doivent être réalisés par les élèves en vue du développement des compétences prescrites? Ont-ils les connaissances scientifiques, disciplinaires et didactiques nécessaires à ce processus? Sont-ils suffisamment outillés et encadrés?

5. Rey (1998) dans la documentation francophone, et Burchell (1995) et Norris (1991) du côté anglophone ont mis en évidence une catégorisation du concept de compétence selon trois modèles distincts: les compétences comportementales, les compétences de fonction, les compétences escientes. Ces trois modèles se situent sur un continuum allant des compétences comportementales, ou behaviorist competence, aux compétences escientes ou interactive model of competence ou encore cognitive constructs of competence. Entre ces deux extrémités se situent les compétences de fonction ou generic competences. Si le premier modèle est fortement influencé par le courant béhavioriste, le deuxième est d'inspiration néobéhavioriste et le troisième, constructiviste. 
Par ailleurs, il faut reconnaître qu'un certain nombre d'incohérences demeurent dans les documents officiels (Araújo-Oliveira, 2010; Lebrun, 2014). Tout d'abord, alors que la construction d'une représentation du temps de l'espace et de la société constitue une compétence importante à développer au $1^{\mathrm{er}}$ cycle du primaire et à laquelle un nombre important de savoirs essentiels liés aux concepts de société, de temps et d'espace y sont rattachés (unités de mesure du temps, utilisation de la ligne du temps et du calendrier, localisation, points de repère, points cardinaux, orientation, lecture de plans simple, de maquettes, initiation à quelques éléments de base de la carte du monde et du Québec, droits et devoirs, métiers, éléments humains, éléments naturels, etc.), on est frappé de constater qu'aucun temps n'est réservé explicitement dans le régime pédagogique du $1^{\mathrm{er}}$ cycle du primaire aux apprentissages SHS ${ }^{6}$ (Gouvernement du Québec, 2018). Il est tout aussi surprenant que tant la compétence à développer que les apprentissages prescrits pour ce cycle ne font pas l'objet d'une évaluation systématique (Gouvernement du Québec, 2009, 2011). D'ailleurs, cette compétence est pratiquement évacuée dans la Progression des apprentissages (Gouvernement du Québec, 2009), qui constitue un complément aux programmes disciplinaires et qui vise à apporter des précisions sur les connaissances que les élèves doivent acquérir et être capables d'utiliser chaque année du primaire, et qui vise aussi à aider les enseignants dans la planification de leur enseignement et des apprentissages que feront leurs élèves. Or, étant donné que cette discipline est considérée comme secondaire en milieu scolaire (AraújoOliveira, Lenoir, Morales-Gómez et McConnell, 2011) et que, pour diverses raisons, il semblerait qu'elle n'ait pas toujours été réellement enseignée au Québec (Lenoir, 2014; Martineau et Soulières, 1991; Conseil supérieur de l'éducation, 1982), le développement de cette première compétence risque d'être largement oblitéré, voire n'être utilisé que comme prétexte aux apprentissages dits de base (lire, écrire et compter). Cela pourrait, par conséquent, réduire largement sa portée sur le développement des compétences envisagées aux $2^{\mathrm{e}}$ et $3^{\mathrm{e}}$ cycles.

De plus, si la perspective comparative (lectures diachronique et synchronique) proposée pour le $2^{\mathrm{e}}$ et $3^{\mathrm{e}}$ cycle du primaire présuppose de s'appuyer sur les résultats de l'analyse descriptive/interprétative des sociétés à comparer, il est pour le moins discutable que les connaissances spécifiques à certaines sociétés ne soient pas identifiées dans la liste des savoirs essentiels (Gouvernement du Québec, 2001) ni dans la progression des apprentissages (Gouvernement du Québec, 2009). Ainsi, on compare la Nouvelle-France de 1645 à celle de 1745 sans que cette dernière soit analysée à partir des mêmes paramètres physiques et humains que la première. On compare aussi la société iroquoienne vers 1500 avec la société inca à la même époque sans qu'une analyse en profondeur des caractéristiques essentielles de la société inca ne soit explicitement proposée. Force est de constater également que, malgré le fait que les compétences prévues pour les $2^{\mathrm{e}}$ et $3^{\mathrm{e}}$ cycles du primaire soient centrées sur

6. Répartition des 25 heures hebdomadaires d'enseignement au $1^{\text {er }}$ cycle: Français (9 heures); Mathématiques (7 heures); Éducation physique et à la santé (2 heures); Anglais, Éthique et culture religieuse, et Arts (7 heures réparties selon le choix de l'enseignant). 
la capacité de l'élève à raisonner et à argumenter, les orientations pour l'évaluation (Ibid.) vont se centrer davantage sur la description de connaissances factuelles. À titre d'exemple, en lien avec l'étude de la société iroquoienne en 1500, on mentionne qu'à la fin du $2^{\mathrm{e}}$ cycle, l'élève doit par lui-même indiquer le mode de vie de cette société (sédentaire) ainsi que nommer des activités économiques propres à cette société (agriculture, chasse, pêche, cueillette et troc, etc.) sans pour autant évaluer la capacité de l'élève à faire des liens entre ces éléments de façon à expliquer et justifier les causes qui amèneraient une société à se sédentariser progressivement (ex. : terres fertiles, développement de l'agriculture et surplus de nourriture, etc.).

\section{ZONE D'OMBRE 4: LES SHS AU PRIMAIRE ET LA DÉMARCHE D'APPRENTISSAGE}

Après avoir précisé le sens des compétences visées, le curriculum de SHS mentionne que pour les développer, l'élève doit être engagé dans une démarche que l'on définit comme une démarche de recherche et de traitement de l'information en géographie et en histoire (Gouvernement du Québec, 2001). Celle-ci est constituée des étapes suivantes: a) la prise de conscience d'un problème (définition d'un problème et élaboration des stratégies de recherche en ayant pour base les connaissances antérieures); b) le questionnement (formulation, organisation et sélection des questions pertinentes); c) planification d'une recherche (établissement d'un plan, construction des outils de collecte de données); d) collecte et traitement de l'information (collecte et classification des données en catégories, comparaison des données en portant un regard critique sur celles-ci); e) organisation de l'information après le traitement; f) communication des résultats sous la forme d'une présentation en utilisant différents supports.

Toutefois, bien que la démarche proposée soit plus structurée que dans les programmes précédents, la description de ses différentes étapes et des actions qui les sous-tendent relève plutôt d'une liste énumérative d'opérations qui portent davantage sur les processus extérieurs (énoncer des questions, repérer des sources d'information, collecter les données, concevoir un plan, etc.) que sur les processus cognitifs qu'elle sous-tend. Par ailleurs, l'appel à une problématisation semble renvoyer à une modalité d'accession au savoir où le problème constitue le point de départ de l'apprentissage (Rey, 2001, 2008). Néanmoins, le recours à la notion de problème souffre d'imprécisions dans les orientations. D'abord, le terme est utilisé dans différents contextes sans que ses attributs ne soient définis (Lebrun, Hasni et AraújoOliveira, 2007). De plus, tantôt on parle de "prendre connaissance d'un problème», ce qui présuppose un problème défini d'avance par l'enseignant ou par le manuel scolaire et soumis par la suite aux élèves, tantôt on mentionne qu'il faut «définir le problème", ce qui laisse entrevoir que celui-ci doit être posé et construit par les élèves, assistés de l'enseignant. Même si l'on considère avec Gérin-Grataloup, Solonel et Tutiaux-Guillon (1994) qu'une problématisation suppose a priori la circonscription 
d'un problème et l'identification de ses éléments constitutifs, il reste qu'on ne sait pas exactement de quel type de problème il est question. S'agirait-il d'un problème d'ordre scientifique qui renverrait aux débats théoriques qui traversent les disciplines de référence? S'agirait-il d'un problème social renvoyant aux problématiques diverses auxquelles les acteurs sociaux sont directement impliqués? Ou s'agirait-il plutôt d'un problème personnel et qui renverrait au vécu des élèves?

La démarche proposée soulève également des interrogations quant au recours aux connaissances antérieures de l'élève en ce sens que celles-ci deviennent le point de départ de la problématisation, mais qu'aucune confrontation entre les connaissances antérieures et les nouvelles n'est préconisée. Dans le même sens, la démarche se termine par la communication des résultats obtenus en utilisant des mots et supports appropriés sans qu'un retour réflexif, à la fois sur la démarche réalisée et sur les résultats obtenus, soit mis en œuvre et sans qu'il y ait eu de véritables synthèses des données et des apprentissages. En effet, il nous semble que la démarche préconisée ne conduit l'élève qu'à des savoirs préexistants dans la mesure où les conceptions initiales, lorsqu'elles sont évoquées au début de la démarche, ne sont jamais convoquées par la suite, et où l'interrogation du sujet sur ses stratégies, les moyens utilisés et les résultats obtenus ne font pas partie du processus.

Enfin, encore qu'elle soit présentée comme étant essentielle au développement des compétences disciplinaires, la démarche préconisée semblerait, selon Lebrun (2014), subordonnée soit à l'autonomie professionnelle des enseignants, soit à une «démarche pédagogique» qui se veut commune à l'ensemble des disciplines scolaires. Comme l'indique l'auteur, d'une part, le discours officiel préconise une participation active de l'élève, tout en laissant à l'enseignant le choix des méthodes et approches pédagogiques à privilégier selon le contexte, la nature des apprentissages et les particularités des élèves: "enseignement magistral, enseignement explicite, enseignement par objectifs, enseignement par démarche inductive, pédagogie par projet, apprentissage coopératif, enseignement stratégique, etc.» (Gouvernement du Québec, 2005a, p. 9.) D'autre part, tant la structuration proposée par le ministère de l'Éducation pour le déroulement d'une situation d'apprentissage et d'évaluation (Gouvernement du Québec, 2005b) que les critères d'évaluation et d'approbation ministériels pour les manuels scolaires (Gouvernement du Québec, 2004, 2007) requièrent que les situations d'enseignement et d'apprentissage (SEA) proposées aux élèves soient structurées selon les trois temps de la démarche pédagogique: préparation, réalisation et intégration des apprentissages. Selon Lebrun (2014, p. 113), en ce sens,

si la priorité accordée à l'uniformisation [...] des SEA dans les manuels scolaires ne signifie nullement que les démarches d'apprentissage spécifiques à chaque discipline scolaire ne sont pas prises en considération, elle ne les situe pas au cœur de l'intervention éducative. 
Il en est de même de la multiplicité des approches pédagogiques listées. Certes, elles ne sont pas forcément incompatibles avec la démarche d'apprentissage prônée pour l'enseignement des SHS. Cependant, cette diversité d'approches centrées tantôt sur l'enseignement (magistrocentrées) tantôt sur les élèves (pédocentrées) (Chamberland, Lavoie et Marquis, 1995) et associées à des courants de pensée fort divergents (Raby et Viola, 2016; Vienneau, 2017) n'est pas mise en relation avec les processus cognitifs de construction des connaissances propre à l'approche constructiviste revendiquée. Tout au contraire, c'est la participation active de l'élève qui est surtout évoquée alors qu'aucune précision quant à l'objet ou à la dimension sur lequel ou laquelle doit porter l'activité cognitive de l'élève n'est fournie dans la documentation ministérielle. Dès lors, on se questionne sur la dimension essentielle devant structurer cette démarche: «[...] est-ce la dimension didactique qui renvoie aux savoirs à enseigner et au processus d'enseignement spécifiques aux différentes disciplines scolaires ou la dimension psychopédagogique qui renvoie aux caractéristiques des élèves et des formules pédagogiques appropriées?»(Lebrun, 2014, p. 113.)

\section{CONSIDÉRATIONS CONCLUSIVES}

Comme nous venons de le voir à travers cette analyse certes non exhaustive, le discours officiel sur les pratiques d'enseignement commande plusieurs ruptures en ce qui a trait à l'enseignement des SHS au primaire: l'adhésion à une approche par compétences, la contribution à la triple mission de l'école, une vision du processus enseignement-apprentissage d'obédience constructiviste, une démarche d'apprentissage structurée et rigoureuse, etc. Toutefois, face aux nombreux questionnements soulevés dans le cadre de cet article, nous ne pouvons pas ignorer l'effet inévitable de la mise en place de telles orientations ministérielles à la fois sur l'interprétation que font les enseignants ou enseignantes et les futurs enseignants ou futures enseignantes de ces orientations que sur les pratiques qu'ils mettront en œuvre.

En fait, le caractère ambigu, incohérent et parfois implicite des orientations peut ainsi influencer largement l'interprétation que font les différents acteurs du milieu éducatif et représenter des obstacles susceptibles d'entraver la mise en œuvre d'une intervention éducative en lien avec les fondements à la base de l'enseignement des SHS. Dès lors, les enseignants peuvent, comme l'affirment Wilson et Berne (1999), réagir et résister aux changements prescrits par le programme en cristallisant des pratiques traditionnelles et en créant des obstacles pour l'émergence de pratiques renouvelées. Ces ambiguïtés et incohérences sont-elles identifiées également dans les discours et pratiques des enseignants ou enseignantes et des futurs enseignants ou futures enseignantes? Sont-elles prises en considération dans le cadre de la formation initiale et continue des enseignants ou enseignantes? Comment s'en sortent ces derniers ou ces dernières dans leurs pratiques effectives, malgré cette marée d'incohérences et d'imprécisions qui traverse les pratiques prescrites matérialisées dans le discours officiel sur la pratique d'enseignement en SHS? Quel statut et quelle place 
les enseignants accordent-ils aux savoirs dans leur enseignement? Quels sont les savoirs privilégiés? Quelles sont les visées éducatives que poursuivent les enseignants dans les relations d'enseignement-apprentissage qu'ils mettent en œuvre? À quels situations, dispositifs et démarches recourent-ils? Comment mettent-ils en œuvre ces situations, dispositifs et démarches?

Certes, les résultats de nos recherches en lien avec les pratiques déclarées et effectives de futurs enseignants ou enseignantes (Araújo-Oliveira, 2010, 2012, 2018; Lebrun, Araújo-Oliveira et Lenoir, 2010) apportent déjà quelques pistes de réponses à ces nombreuses interrogations. Ces recherches constatent notamment le caractère bien enraciné de certaines convictions sur l'enseignement et l'apprentissage des SHS chez les futurs enseignants et la grande difficulté que rencontrent les dispositifs de formation à faire évoluer ces convictions. Ils laissent entrevoir que les pratiques d'enseignement chez les futurs enseignants ou enseignantes en histoire et en géographie, ainsi que le discours qu'ils entretiennent sur leurs pratiques, restent encore centrés sur des aspects factuels de type dénomination-localisation, sans développer d'apprentissages véritablement conceptuels. Toutefois, la réflexion réalisée dans le cadre de cet article met en exergue la nécessité pour la recherche en didactique des SHS de s'attarder à comprendre les pratiques d'enseignement afin de produire des connaissances qui puissent, entre autres: a) clarifier la raison d'être de l'introduction de cette discipline dans un curriculum de formation au primaire (passe-temps, développement identitaire, développement de la pensée critique, etc.); b) clarifier les processus cognitifs par lesquels les élèves construisent les savoirs historiques et géographiques; et c) revisiter les prescriptions et orientations ministérielles afin d'éliminer le plus possible ses ambiguïtés et ainsi mieux outiller les différents acteurs du milieu éducatif.

\section{Références bibliographiques}

ARAÚJO-OLIVEIRA, A. (2010). Caractéristiques des pratiques d'enseignement en sciences humaines chez de futurs enseignants du primaire en contexte de stage (Thèse de doctorat). Université de Sherbrooke.

ARAÚJO-OLIVEIRA, A. (2012). Finalités éducatives sous-jacentes aux pratiques des futurs enseignants du primaire: le cas de l'enseignement des sciences humaines et sociales au Québec. Phronesis, 1(4), 84-97. 
ARAÚJO-OLIVEIRA, A. (2018). Le processus de problématisation dans l'enseignement des sciences humaines et sociales au primaire. Dans A. Araújo-Oliveira, I. Chouinard et G. Pellerin (dir.), L'analyse des pratiques professionnelles dans les métiers relationnels: perspectives plurielles (p. 187-210). Québec: Presses de l’Université du Québec.

ARAÚJO-OLIVEIRA, A., LENOIR, Y., MORALES-GÓMES, A. et McCONNELL, A.C. (2011). Práticas interdisciplinares no ensino primário : concepções de professores e futuros professores no Québec. Educação e sociedade, 32(117), 1125-1145.

AUDIGIER, F. (1999). Les représentations de la géographie dans l'enseignement primaire en France: habitat commun, voisinage et distances. Cahier de géographie du Québec, 43(120), 395-412.

BRONCKART, J.-P. et Dolz, J. (2002). La notion de compétence: quelle pertinence pour l'étude de l'apprentissage des actions langagières? Dans J. Dolz et E. Ollagnier (dir.), L'énigme de la compétence en éducation (p. 27-44). Bruxelles: De Boeck.

BURCHELL, H. (1995). A usefull role for competence statements in post-compulsory teacher education? Assessment and Evaluation in Higher Education, 20(3), 251-259.

CAILLOT, M. (1994). Des objets aux compétences dans l'enseignement scientifique: une évolution de vingt ans. Dans F. Ropé et L. Tanguy (dir.), Savoirs et compétences. De l'usage de ces notions dans l'école et dans l'entreprise (p. 94-117). Paris: L'Harmattan.

CASTAÑON, G. A. (2007). Constructivismo, inatismo e realismo: compatíveis e complementares. Ciências e cognição, 10, 115-131.

CHAMBERLAND, G., LAVOIE, L. et MARQUIS, D. (1995). 20 formules pédagogiques. Sainte-Foy: Presses de l’Université du Québec.

COLL, C. (2003). Entrevista a Faoze Chibli. Revista educação, 7(78), (n.p).

COLL, C., MARTÍN, E., MAURI, T. et MIRAS, M. (1993). El constructivismo en el aula. Madrid: Graó.

CONSEIL SUPÉRIEUR DE L'ÉDUCATION (1982). Le sort des matières dites secondaires au primaire. Avis au Ministère de l'éducation. Québec: Conseil supérieur de l'éducation.

DAUDEL, C. (1990). Les fondements de la recherche en didactique de la géographie. Berne: Peter Lang. 
GAYA, A. (2006). A reinvenção dos corpos: Por uma pedagogia da complexidade. Sociologias, 8(15), 250-272.

GÉRIN-GRATALOUP, A.-M., SOLONEL, M. et TUTIAUX-GUILLON, N. (1994). Situations-problèmes et situations scolaires en histoire-géographie. Revue française de pédagogie, 106, 25-37.

GOUVERNEMENT DU QUÉBEC (1971). Orientation nouvelle des sciences humaines à l'élémentaire. Québec: Ministère de l'éducation.

GOUVERNEMENT DU QUÉBEC (1981). Programme d'études - primaire: sciences humaines (histoire, géographie, vie économique et culturelle). Québec: Ministère de l'éducation.

GOUVERNEMENT DU QUÉBEC (1983). Guide pédagogique - primaire: sciences humaines (histoire, géographie, vie économique et culturelle). Québec: Ministère de l'éducation.

*GOUVERNEMENT DU QUÉBEC (1996). Rénover notre système d'éducation: dix chantiers prioritaires. Rapport final de la commission des États généraux sur l'éducation. Québec: Ministère de l'éducation.

*GOUVERNEMENT DU QUÉBEC (1997a). Prendre le virage du succès: Plan d'action pour la réforme de l'éducation. Québec: Ministère de l'éducation.

*GOUVERNEMENT DU QUÉBEC (1997b). L'école, tout un programme. Énoncé de politique éducative. Québec: Ministère de l'éducation.

*GOUVERNEMENT DU QUÉBEC (2001). Programme de formation de l'école québécoise. Éducation préscolaire et enseignement primaire. Québec: Ministère de l'éducation.

*GOUVERNEMENT DU QUÉBEC (2004). Évaluation des aspects pédagogiques du matériel éducatif: primaire et secondaire. Québec: Ministère de l'éducation.

*GOUVERNEMENT DU QUÉBEC (2005a). Le renouveau pédagogique. Ce qui définit le changement. Préscolaire, primaire et secondaire. Québec: Ministère de l'Éducation, du Loisir et du Sport.

*GOUVERNEMENT DU QUÉBEC (2005b). Cadre de référence en évaluation des apprentissages au secondaire. Québec: Ministère de l'éducation, du loisir et du sport.

*GOUVERNEMENT DU QUÉBEC (2007). L'approbation du matériel didactique. Québec: Ministère de l'éducation, du loisir et du sport. 
*GOUVERNEMENT DU QUÉBEC (2009). Progression des apprentissages : Géographie, histoire et éducation à la citoyenneté. Québec: Ministère de l'éducation, du loisir et du sport.

*GOUVERNEMENT DU QUÉBEC (2011). Cadre d'évaluation des apprentissages: Géographie, histoire et éducation à la citoyenneté. Québec: Ministère de l'éducation, du loisir et du sport.

*GOUVERNEMENT DU QUÉBEC (2018). Régime pédagogique de l'éducation préscolaire, de l'enseignement primaire et de l'enseignement secondaire. Repéré à http://legisquebec.gouv.qc.ca/fr/pdf/cr/l-13.3,\%20R.\%208.pdf

JONNAERT, P. (2002). Compétences et socioconstructivisme: un cadre théorique. Bruxelles: De Boeck.

JONNAERT, P. (2006). Constructivisme, connaissances et savoirs. Transfert, 3.

JONNAERT, P., BARRETTE, J., BOUFRAHI, S. et MASCIOTRA, D. (2004). Contribution critique au développement des programmes d'études: compétences, constructivisme et interdisciplinarité. Revue des sciences de l'éducation, XXX(3), 667-696.

LAROSE, F., LENOIR, Y., PEARSON, M. et MORIN, J.-F. (2005). Les construits de compétence et de transversalité dans le nouveau curriculum du primaire et du secondaire: fondements épistémologiques et perspectives. Dans Y. Lenoir, F. Larose et C. Lessard (dir.), Le curriculum de l'enseignement primaire: regards critiques sur ses fondements et ses lignes directrices (p. 139-165). Sherbrooke: Éditions du CRP.

LAURIN, S. (1998). La relation espace-temps dans la formation à l'univers social. Dans J.L. Klein et S. Laurin (dir.), L'éducation géographique: formation du citoyen et conscience territoriale (p. 9-31). Québec: Presses de l'Université du Québec.

LAVILLE, C. (1991). L'épistémologie n'est peut-être pas absolument nécessaire pour enseigner les sciences humaines au primaire, mais ça aide joliment. Dans Y. Lenoir et M. Laforest (dir.), L'enseignement des sciences humaines au primaire: développement, sous-développement ou développement du sous-développement? (p. 5-66). Sherbrooke: Éditions du CRP.

LE ROUX, A. (2005). Didactique de la géographie ( $3^{\mathrm{e}}$ éd.). Caen: Presses universitaires de Caen (1 $1^{\text {re }}$ éd. 1997).

LEBRUN, N. (1993). Importance des sciences humaines au niveau primaire. Traces, 31(5), 1822. 
L'intervention éducative en sciences humaines et sociales au primaire à travers les zones d'ombre des discours sur les pratiques d'enseignement

LEBRUN, J. (2009). Les compétences, concepts et savoirs essentiels à développer. Dans J. Lebrun et A. Araújo-Oliveira (dir.), Lintervention éducative en sciences humaines au primaire: des fondements aux pratiques (p. 66-92). Montréal: Chenelière éducation.

LEBRUN, J. (2014). Les conceptions disciplinaires véhiculées dans le discours des futurs enseignants et des manuels scolaires. La survalorisation de la mise en activité de l'élève. Dans Larouche, M.-C. et A. Araújo-Oliveira (dir.). Les sciences humaines à l'école primaire québécoise. Regards croisés sur un domaine de recherche et d'intervention (p. 107-124). Québec: Presses de l'Université du Québec.

LEBRUN, J. et ARAÚJO-OLIVEIRA, A. (2010). La introducción del concepto de competencia en el análisis didáctico de las prácticas de enseñanza: el caso de la enseñanza de Ciencias Humanas en la educación básica de Quebec. Pensamiento educativo, 44-45, 211-235.

LEBRUN, J., ARAÚJO-OLIVEIRA, A. et LENOIR, Y. (2010). L'enseignementapprentissage des sciences humaines: quelles finalités, quelles difficultés et quelles compétences professionnelles? Résultats d'une enquête auprès de futurs enseignants québécois du primaire. Revue canadienne de l'éducation, 33(1), $1-30$.

LEBRUN, J., HASNI, A. et ARAÚJO-OLIVEIRA, A. (2007, octobre). Situation-problème et savoirs scolaires disciplinaires: la difficile jonction entre le social, l'individuel et l'épistémologique dans l'enseignement primaire québécois des sciences et des sciences humaines. Communication présentée aux dixièmes rencontres internationales du Réseau de recherche en éducation et en formation (REF), Sherbrooke.

LEBRUN, J., HASNI, A. et OLIVEIRA, N. (2008). Point de vue de futurs enseignants québécois du primaire sur les programmes des sciences et technologie et des sciences humaines et leur enseignement. Dans G. Baillat et A. Hasni (dir.), L'école primaire et les savoirs scolaires: perspectives actuelles (p. 219-247). Sherbrooke: Édition du CRP.

LENOIR, Y. (1989). Apport spécifique des sciences humaines dans la formation générale des écoliers du primaire. Dans G.-R. Roy (dir.), Actes $d u 2^{e}$ Congrès des sciences de l'éducation de langue française du Canada (p. 681-691). Sherbrooke: Éditions du CRP.

LENOIR, Y. (1993). Entre Descartes et Hegel: de quels sens peut-il être question en didactique? Contribution au développement d'une épistémologie de la didactique. Dans P. Jonnaert et Y. Lenoir (dir.), Sens des didactiques et didactique du sens (p. 29-99). Sherbrooke: Éditions du CRP. 
LENOIR, Y. (1996). Médiation cognitive et médiation didactique. Dans C. Raisky et M. Caillot (dir.), Au-delà des didactiques, le didactique: débats autour de concepts fédérateurs (p. 223-251). Bruxelles: De Boeck.

LENOIR, Y. (2014). Les sciences humaines au primaire face à la déliquescence de l'humain et du social, une reconceptualisation épistémologique des fondements. Dans M.-C. Larouche et A. Araújo-Oliveira (dir.), Les sciences humaines à l'école primaire québécoise (p. 301-327). Québec: Presses de l’Université du Québec.

LENOIR, Y. et LAROSE, F. (2005). Analyse de la perspective constructiviste du nouveau curriculum québécois de l'enseignement primaire. Dans Y. Lenoir, F. Larose et C. Lessard (dir.), Le curriculum de l'enseignement primaire: regards critiques sur ses fondements et ses lignes directrices (p. 109-140). Sherbrooke: Éditions du CRP.

LENOIR, Y., LAROSE, F., BIRON, D., ROY, G.-R. et SPALLANZANI, C. (1999). Le concept de compétence dans la formation à l'enseignement primaire au Québec. Recherche et formation, 30, 143-163.

LIBRESCO, A.S. (2003). Current events matters... for elementary social studies methods students. The international social studies forum, 3(1), 273-276.

MARTINEAU, R. et SOULIÈRES, R. (1991). La situation de l'enseignement des sciences humaines dans le milieu scolaire: 1980-1990. Dans Y. Lenoir et M. Laforest (dir.), L'enseignement des sciences humaines au primaire: développement, sous-développement ou développement du sous-développement? (p. 31-43). Sherbrooke: Éditions du CRP.

MONIOT, H. (1993). Didactique de l'histoire. Paris : Éditions Nathan.

NORRIS, N. (1991). The trouble with competence. Cambridge journal of education, 21(3), 331341.

PERRENOUD, P. (2002). D'une métaphore à l'autre: transférer ou mobiliser ses connaissances? Dans J. Dolz, et E. Ollagnier (dir.), L'énigme de la compétence en éducation (p. 45-60). Bruxelles: De Boeck.

RABY, C. et VIOLA, S. (dir.). (2016). Modèles d'enseignement et théories d'apprentissage: de la théorie à la pratique ( $2^{\mathrm{e}}$ éd.). Anjou: Les éditions CEC.

REY, B. (1998). Les compétences transversales en question ( $2^{\mathrm{e}}$ éd.). Paris: ESF ( $1^{\mathrm{re}}$ éd. 1996).

REY, B. (2001). Manuels scolaires et dispositifs didactiques. Dans Y. Lenoir, B. Rey, G.R. Roy et J. Lebrun (dir.), Le manuel scolaire et l'intervention éducative. Regards critiques sur ses apports et ses limites (p. 25-40). Sherbrooke: Éditions du CRP. 
REY, B. (2008). Faire la classe à l'école élémentaire (6e éd.). Paris: ESF (1 ${ }^{\mathrm{re}}$ éd. 1998).

TUTIAUX-GUILLON, N. (2015). Questions socialement vives et recomposition disciplinaire de l'histoire-géographie: entre opportunités et résistances. Dans F. Audigier, A. Sgard et N. Tutiaux-Guillon (dir.), Sciences de la nature et de la société dans une école en mutation: fragmentations, recompositions, nouvelles alliances? (p. 139-150). Belgique: Éditions de Boeck.

VIENNEAU, R. (2017). Apprentissage et enseignement: théories et pratiques ( $3^{\mathrm{e}}$ éd.). Montréal: Gaëtan Morin éditeur.

WERNECK, V. R. (2006). Sobre o processo de construção do conhecimento: O papel do ensino e da pesquisa. Ensaio: avaliação e políticas públicas em educação, 14(51), 173196.

WILSON, S.M. et BERNE, J. (1999). Teaching learning and the acquisition of professional knowledge: An examination of research on contemporary professional development. Review of research in education, 24, 173-209. 